\title{
Molecular epidemiology and antimicrobial resistance of group a streptococcus recovered from patients in Beijing, China
}

\author{
Hongxin Li ${ }^{1 *} \mathbb{D}$, Lin Zhou², Yong Zhao ${ }^{3}$, Lijuan $\mathrm{Ma}^{2}$, Xiaoyan Liu and Jin $\mathrm{Hu}^{1 *}$
}

\begin{abstract}
Background: Group A streptococcus (GAS) is an important human pathogen responsible for a broad range of infections. Epidemiological surveillance has been crucial to detect changes in the geographical and temporal variation of the disease pattern. The objective of this study was to investigate the molecular epidemiological characteristics and antimicrobial resistance of GAS isolates from patients in Children's Hospital in Beijing.
\end{abstract}

Methods: From 2016 to 2017, pharyngeal swab samples were collected from the outpatients in Children's Hospital, Capital Institute of Pediatrics, who were diagnosed with scarlet fever. Antimicrobial susceptibility test was performed according to the distribution of conventional antibiotics and Clinical and Laboratory Standards Institute (CLSI) recommendations. The distribution of the macrolide-resistance genes (ermB, ermA, mefA), emm ( $M$ proteincoding gene) typing, and superantigens (SAg) gene profiling were examined by polymerase chain reaction (PCR).

Results: A total of 297 GAS isolates were collected. The susceptibility of the isolates to penicillin, ceftriaxone, and levofloxacin was 100\%. The resistance rate to erythromycin and clindamycin was 98.3 and $96.6 \%$, respectively. The dominant emm types were emm12 (65.32\%), emm1 (27.61\%), emm75 (2.69\%), and emm89 (1.35\%). Of the 297 isolates, 290 (97.64\%) carried the ermB gene, and 5 (1.68\%) carried the mefA gene, while none carried the ermA gene. The most common superantigen genes identified from GAS isolates were smeZ (96.97\%), speC (92.59\%), speG (91.58\%), ssa (85.52\%), spel (54.55\%), speH (52.19\%), and speA (34.34\%). Isolates with the genotype emm1 possessed speA, speC, speG, speJ, speM, ssa, and smeZ, while emm12 possessed speC, speG, speH, spel, speM, ssa, and smeZ superantigens.

Conclusions: The prevalent strain of GAS isolates in Beijing has a high resistance rate to macrolides; however, penicillin can still be the preferred antibiotic for treatment. Erythromycin resistance was predominantly mediated by ermB. The common emm types were emm12 and emm1. There was a correlation between emm and the superantigen gene. Thus, long-term monitoring and investigation of the emm types and superantigen genes of GAS prevalence are imperative.

Keywords: Group a streptococcus, GAS, Streptococcal M protein, emm types, Superantigens, Antimicrobial resistance, Scarlet fever, Children

\footnotetext{
*Correspondence: lihxin@126.com; hujin1970@sohu.com

'Department of Dermatology, Children's Hospital Affiliated to Capital Institute of Pediatrics, Beijing 100020, China

Full list of author information is available at the end of the article
} 


\section{Background}

Streptococcus pyogenes (Lancefield group A streptococcus; GAS) is a major pathogen causing infectious diseases in children. It causes suppurative and nonsuppurative diseases, such as erysipelas, suppurative tonsillitis, scarlet fever, rheumatic fever, and glomerulonephritis [1]. Globally, there are about 616 million cases of GAS pharyngitis every year, among which, 17,800 cases are new infections, and about 517,000 patients with severe GAS are deceased every year [2]. Recently, the positive rate of GAS was estimated at $21.2 \%$ in the pharyngeal culture of patients diagnosed as "streptococcal infection/tonsillitis/angina" [3]. Moreover, the incidence of streptococcal pharyngitis is common in children aged 0-14 years. From 2012 to 2014, the average number of positive cases of streptococcal culture was 2685.1/100,000 children in Beijing, including 1652.7 outpatient visits [4]. In 2011, scarlet fever broke out in mainland China and Hong Kong, with a sharp increase in incidence $[5,6]$. Penicillin is the preferred clinical treatment of GAS infection, while erythromycin is the first alternative antibiotic for patients allergic to penicillin, followed by clindamycin. The resistance rate to macrolidesis gradually increasing, which might be related to the overuse of such antibiotics $[7,8]$. In our previous study, 95 isolates were recovered from suppurative tonsillitis, acute glomerulonephritis, scarlet fever and streptococcal dermatitis. The resistance rates of the isolates to erythromycin, clindamycin and tetracycline were 98.9, 100, $94.7 \%$ respectively [9].

The M protein encoded by the $\mathrm{emm}$ gene is the main pathogenic factor of GAS, and different types vary in pathogenicity. Therefore, emm typing is employed to track outbreak and routinely monitor GAS diseases. From 2011 to 2013, the proportion of emm12.0 in children with GAS infection in Xicheng District of Beijing decreased gradually, and emm1.0 increased every year [10]. The interplay among GAS diseases, emm types, superantigen gene, and antimicrobial resistance needs further investigation [11, 12]. The resistance of GAS to macrolides is related to the mechanism underlying target modification mediated by ermA and ermB and the pumping mechanism mediated by mefA. Intriguingly, different primary mechanisms of resistance regulate various epidemic strains [13]. The superantigen genes are the main virulence factors and closely related to the pathogenicity of GAS. Hitherto, 11 superantigen genes, including speA, speC, speG, speH, speI, speJ, speK, speL, speM, smeZ, and ssa, were found to be distributed among various strains $[14,15]$. Previously, the emm typing and superantigen distribution of GAS strains from different regions of mainland China were investigated, which indicated that the typing and antimicrobial resistance were slightly different $[16,17]$. In this study, we recovered GAS isolates from patients with scarlet fever in Children's Hospital, Capital Institute of Pediatrics from 2016 to 2017, and conducted antimicrobial susceptibility test, emm genotype analysis, and combined analysis of superantigen to assess the molecular epidemiological characteristics and antimicrobial resistance mechanisms of GAS strains.

\section{Methods}

\section{Strain collection}

A total of 297 cases of GAS isolates were recovered from pediatric patients presenting scarlet fever in the Children's Hospital, Capital Institute of Pediatrics, from January 2016 to December 2017. Throat swabs were obtained from patients by two physicians for routine microbiologic analysis.

\section{Bacterial identification}

The throat swab samples were inoculated on a Columbia blood plate (BD, USA) and cultured in a $\mathrm{CO}_{2}$ incubator at $37^{\circ} \mathrm{C}$ for $24-36 \mathrm{~h}$. A single round colony with the transparent hemolytic ring was selected, cultured, and evaluated by Gram staining. The Streptococcus Grouping Kit (Oxoid, Basingstoke, UK) was used to classify the suspicious colonies.

\section{Antimicrobial susceptibility testing}

According to the distribution of conventional antibiotics and recommendations of Clinical and Laboratory Standards Institute (CLSI), disk diffusion method (K-B method) was used to test the susceptibility of the isolated Streptococcus pyogenes to ten antibiotics. The distance between each disk was $>24 \mathrm{~mm}$, and the distance between the center of the disk and edge of the dish was $>15 \mathrm{~mm}$. The plate was incubated at $37^{\circ} \mathrm{C}$ for $18-24 \mathrm{~h}$ and only two plates were stacked. The susceptibility of bacteria was determined by the diameter of the inhibition growth and CLSI standard [18]. Streptococcus pneumoniae ATCC 49619 was used as a control strain..

\section{DNA extraction of GAS genome}

DNA extraction of GAS genome was performed according to the recommended method by the Center for Disease Control and Prevention (CDC; https://www.cdc. gov/streplab/groupa-strep/emm-typing-protocol.html), one ring of GAS was suspended in $300 \mu \mathrm{L}$ saline and incubated at $70{ }^{\circ} \mathrm{C}$ for $15 \mathrm{~min}$. The precipitate, obtained by centrifugation and suspended in $50 \mu \mathrm{L} \mathrm{TE}(\mathrm{pH} 8.0)$, $10 \mu \mathrm{L}$ mutanolysin $(3000 \mathrm{U} / \mathrm{mL})$, and $2 \mu \mathrm{L}$ hyaluronidase $(30 \mathrm{mg} / \mathrm{mL})$, was reheated at $100^{\circ} \mathrm{C}$ for $10 \mathrm{~min}$ and centrifuged to obtain genomic DNA. 


\section{emm genotyping}

The emm-typing of all isolates was performed according to the protocols and recommendations of the CDC. The sequence data were uploaded to the emm typing database (https://www2.cdc.gov/vaccines/biotech/strepblast. asp) for comparison. A sequence was considered to belong to a specific emm gene when the first $160 \mathrm{nt}$ of the sequence exhibited $>95 \%$ sequence identity with that of the reference $e m m$ gene.

\section{Erythromycin-resistance gene detection}

All GAS isolates were screened for the presence of ermB, $\operatorname{erm} A$, and $m e f A$ as previously reported [19] Primer sequences are listed in Table 1 . The reaction system $(25 \mu \mathrm{L})$ contained $1 \mu \mathrm{L} 2 \mathrm{mM}$ DNA template, $1 \mu \mathrm{L}(10$ $\mathrm{mM})$ for each primer, $0.2 \mu \mathrm{L}$ Taq DNA polymerase $(5 \mathrm{U} /$ $\mu \mathrm{L}), 2 \mu \mathrm{L}$ of $2.5 \mathrm{mM}$ dNTPs, $2.5 \mu \mathrm{L}$ of $10 \times$ Taq buffer (2.5 mM $\mathrm{MgCl}_{2}$ plus; Takara Biotechnology Co.), and $17.3 \mu \mathrm{L}$ water. The initial denaturation was performed for $1 \mathrm{~min}$ at $94^{\circ} \mathrm{C}$, followed by denaturation for $30 \mathrm{~s}$ at $94{ }^{\circ} \mathrm{C}$ annealing for $30 \mathrm{~s}$ at $54{ }^{\circ} \mathrm{C}$, extended for $30 \mathrm{~s}$ at $72{ }^{\circ} \mathrm{C}$ for 29 cycles and final extension for $3 \mathrm{~min}$ at $72{ }^{\circ} \mathrm{C}$.

\section{Superantigen detection}

Eleven superantigen genes (speA, speC, speG, speH, speI, speJ, speK, speL, speM, ssa, and smeZ) were amplified by PCR using the genomic DNA extracted during emm typing as previously described [20]. By comparing the size of the products with the predicted positive fragments, the expression of superantigen in GAS was determined. The primers used for amplification are listed in Table 2.

\section{Results}

Patients' characteristics

297 isolates were recovered from 1183 throat swab samples. For the 297 patients, the mean age was 6.17 years, the median was 6 years (range from 1 to 13 years), and 175 were boys, 122 were girls.

\section{Antimicrobial susceptibility testing results}

All 297 GAS isolates were sensitive to penicillin, ceftriaxone, cefotaxime, cefepime, vancomycin, and levofloxacin. They were resistant to erythromycin, clindamycin and tetracycline with the resistance rate $98.3 \%(292 / 297)$,
96.6\% (287/297) and $90.23 \%(268 / 297)$, respectively (Table 3).

\section{Distribution of emm types}

Overall, $9 \mathrm{emm}$ types were detected in GAS isolates, including 28 subtypes from 2016 to 2017. The majority of the cases were emm12 (65.32\%, 194/297), emm1 (27.61\%, 82/297), emm75 (2.69\%, 8/297), and emm89 (1.35\%, 4/297). emm12.0 and emm1.0 were the most prevalent subtypes, accounted for 46.8 and $26.26 \%$, respectively. A variant subtype (stg485.0) was also detected. The distribution of the emm types is shown in Table 4.

\section{emm types and erythromycin-resistance genes}

Among the 297 isolated GAS strains, 290 (97.64\%) carried $\operatorname{erm} B$, while $5(1.68 \%)$ carried mefA and none carried ermA (Table 5). Three erythromycin sensitive strains were found among the isolates, distributed in subtype emm12.0 and emm3.1. None of the three isolates showed the presence of $\operatorname{erm} A, \operatorname{erm} B$, and $m e f A$. Clindamycin-sensitive strains were distributed in emm12.0 and emm3.1 subtypes. The positive rates of ermB, ermA, and mefA in emm12 and emm1 strains were $45.79,0,0.34$ and $26.3 \%, 0,0.67 \%$, respectively.

\section{emm type and superantigen distribution}

Of 297 isolates, the most common superantigen genes identified from S. pyogenes were smeZ (96.97\%), speC (92.59\%), speG (91.58\%), and ssa (85.52\%), while the expression rate of other superantigens was slightly lower: speI (54.55\%), speH (52.19\%), speA (34.34\%), speM (24.57\%), speJ (22.22\%), speL (5.05\%), and speK (2.02\%). emm1 tended to harbor speA, speC, speG, speJ, speM, ssa, and smeZ, but less speI, speK, and speL. emm12 tended to harbor speC, speG, speH, speI, speM, ssa, and smeZ, with little or no speJ, speK, speL. Variant stg485 did not express any detected superantigens. The details of superantigen distribution are shown in Table 5.

\section{Discussion}

S. pyogenes or GAS is a leading pathogen causing infectious diseases in children. The GAS infection manifests as mild non-invasive diseases, such as acute pharyngitis

Table 1 Primer sequences for erythromycin-resistance genes

\begin{tabular}{lllr}
\hline Gene & Primer direction & Primer sequence & Amplicon size (bp) \\
\hline ermB & Forward & $5^{\prime}$-ATTGGAACAGGTAAAGGGC-3' & 639 \\
& Reverse & 5'-GAACATCTGTGGTATGGCG-3' & 530 \\
ermA & Forward & 5'-AACTTGTGGAAATGAGTCAACGG-3' & 348 \\
& Reverse & Forward & $5^{\prime}$-CAGAATCTACATTAGGCTTAGGG-3' \\
& Reverse & $5^{\prime}$-AGTATCATTAATCACTAGTGC-3' & \\
\hline
\end{tabular}


Table 2 Primers for PCR of virulence and superantigen genes

\begin{tabular}{|c|c|c|c|c|}
\hline Gene & Primer direction & $\begin{array}{l}\text { Primer } \\
\text { sequence }\end{array}$ & $\begin{array}{l}\text { Annealing } \\
\text { Temperature }\left({ }^{\circ} \mathrm{C}\right)\end{array}$ & $\begin{array}{l}\text { Amplicon } \\
\text { Size (bp) }\end{array}$ \\
\hline \multirow[t]{2}{*}{ speA } & Forward & 5'-ATGGAAAACAATAAAAAAGTATTG-3' & 52 & 756 \\
\hline & Reverse & 5'-TTACTTGGTTGTTAGGTAGACTTC-3' & & \\
\hline \multirow[t]{2}{*}{ spec } & Forward & 5'-AATTTCGATTCTGCCGCTTA-3' & 52 & 400 \\
\hline & Reverse & 5'-GCAGGGTAAATITTCAACGAC-3' & & \\
\hline \multirow[t]{2}{*}{ speG } & Forward & 5'-TCATGTGTIITTAGCTATGGAAGTC-3' & 52 & 590 \\
\hline & Reverse & 5'- ACTGTCTCGACTTAAAAGCTTATCA-3' & & \\
\hline \multirow[t]{2}{*}{ speH } & Forward & 5'-AGATTGGATATCACAGG-3' & 52 & 416 \\
\hline & Reverse & 5'- CTATTCTCTCGTTATTGG-3' & & \\
\hline \multirow[t]{2}{*}{ spel } & Forward & 5'- AATGAAGGTCCGCCATTTTC-3' & 52 & 516 \\
\hline & Reverse & 5'-TCTCTCTGTCACCATGTCCTG-3' & & \\
\hline \multirow[t]{2}{*}{ spel } & Forward & 5'-GATAGTGAAAATATTAAAGACG-3' & 52 & 630 \\
\hline & Reverse & 5'- TTATTTAGTCCAAAGGTAAATATC-3' & & \\
\hline \multirow[t]{2}{*}{ spek } & Forward & 5'-GTGTGTCTAATGCCACCGTCT-3' & 52 & 564 \\
\hline & Reverse & 5'-GGAACATATATGCTCCTAGAT-3' & & \\
\hline \multirow[t]{2}{*}{ spel } & Forward & 5'-CAGCACCTTCCTCTTTCTCG-3' & 52 & 459 \\
\hline & Reverse & 5'-GGAAAAAGAGGGACGCAAG-3' & & \\
\hline \multirow[t]{2}{*}{ speM } & Forward & 5'-GGATGAGTGAATAAATCGGTAAAC-3' & 55 & 425 \\
\hline & Reverse & 5'-AGTCTGGGACGATGATAA-3' & & \\
\hline \multirow[t]{2}{*}{ ssa } & Forward & 5'-TGATCAAATATTGCTCCAGGTG-3' & 52 & 502 \\
\hline & Reverse & 5'-TCCACAGGTCAGCTITTACAG-3' & & \\
\hline \multirow[t]{2}{*}{ smez } & Forward & 5'-CTTCAATATTCATTGCAATAATTTC-3' & 52 & 430 \\
\hline & Reverse & 5'-TGTAACTGTTGTTTTGTTAGTTGAT-3' & & \\
\hline
\end{tabular}

or life-threatening invasive diseases, such as sepsis and toxic shock syndrome [15]. Scarlet fever is a acute infectious disease caused by GAS, that can affect people of all ages, but it is most often seen in children. Before the advent of antibiotics, scarlet fever was extremely serious, often causing long periods of illness, many dangerous complications, and even death. A re-emergence of

Table 3 Antimicrobial susceptibility test of 297 isolates of GAS from Children's Hospital during 2016 to 2017

\begin{tabular}{llll}
\hline Antibiotic & \multicolumn{3}{l}{ Susceptibility $(\mathrm{n} / \%)$} \\
\cline { 2 - 4 } & Susceptible & Intermediate & Resistant \\
\hline Penicillin C & $297 / 100$ & 0 & 0 \\
Ceftriaxone & $297 / 100$ & 0 & 0 \\
Clindamycin & $8 / 2.69$ & $2 / 0.67$ & $287 / 96.6$ \\
Erythromycin & $3 / 1.01$ & $2 / 0.67$ & $292 / 98.3$ \\
Tetracycline & $16 / 5.39$ & $13 / 4.38$ & $268 / 90.23$ \\
Vancomycin & $297 / 100$ & 0 & 0 \\
Chloramphenicol & $283 / 95.29$ & $12 / 4.04$ & $2 / 0.67$ \\
cefepime & $297 / 100$ & 0 & 0 \\
cefotaxime & $297 / 100$ & 0 & 0 \\
Levofloxacin & $297 / 100$ & 0 & 0 \\
\hline
\end{tabular}

scarlet fever has been noted in Hong Kong, mainland China, South Korea, and England, UK, and other countries around the world since 2008 to 2014 [21-23]. Penicillin has always been the preferred treatment for the GAS infection. In penicillin allergic patients, macrolides are the most commonly used antibiotics for treating streptococcal infections. However, the resistance rate of macrolides has also been increasing gradually [7]. Of the GAS isolates recovered from the throat swabs of children with pharyngitis in Madison, Wisconsin, 15\% demonstrated nonsusceptibility for clindamycin and erythromycin, and inducible resistance (positive D-test) was detected in $12 \%$ isolates [24]. S. pyogenes isolates collected from infected patients from 7 cities/provinces in China during the years 2009-2016, were phenotypically susceptible to penicillin, ampicillin, cefotaxime, and vancomycin, whereas $93.5,94.2$, and $86.4 \%$ were resistant to erythromycin, clindamycin, and tetracycline, respectively [25]. In this study, the GAS isolates recovered from children with scarlet fever were highly sensitive to penicillin, cephalosporin, levofloxacin, and vancomycin while the resistance rates to erythromycin, clindamycin and tetracycline are 98.3, 96.6 and 90.23\%, respectively (see Table 3). No significant shift was detected in the 
Table 4 Distribution of the emm genotypes of GAS isolates from Children's Hospital during 2016 to 2017

\begin{tabular}{|c|c|c|}
\hline emm types & emm subtypes & Count (n) \\
\hline \multirow[t]{4}{*}{ emm1 } & emm1.0 & 78 \\
\hline & emm 1.25 & 1 \\
\hline & emm1.3 & 1 \\
\hline & emm 1.33 & 2 \\
\hline \multirow[t]{15}{*}{ emm12 } & emm 12.0 & 139 \\
\hline & emm12.12 & 1 \\
\hline & emm12.13 & 1 \\
\hline & emm12.19 & 27 \\
\hline & emm12.20 & 1 \\
\hline & emm12.21 & 2 \\
\hline & emm12.30 & 1 \\
\hline & emm12.36 & 4 \\
\hline & emm12.37 & 8 \\
\hline & emm 12.40 & 2 \\
\hline & emm12.66 & 2 \\
\hline & emm12.69 & 2 \\
\hline & emm12.70 & 1 \\
\hline & emm12.72 & 2 \\
\hline & emm12.76 & 1 \\
\hline \multirow[t]{3}{*}{ emm6 } & emm6.19 & 1 \\
\hline & emm6.4 & 1 \\
\hline & emm6.89 & 1 \\
\hline emm75 & emm75.0 & 8 \\
\hline emm89 & emm89.0 & 4 \\
\hline stg485 & stg 485.0 & 1 \\
\hline emm225 & emm225 & 1 \\
\hline emm3 & emm3.1 & 3 \\
\hline emm4 & emm4.0 & 1 \\
\hline Total & 28 & 297 \\
\hline
\end{tabular}

resistance rate of GAS isolates to antibiotics between 2016 and 2017. These findings were consistent with those from previous study in 2013 that the resistance rates of isolates obtained from scarlet fever in Beijing to erythromycin, clindamycin and tetracycline were 99.3, 99.3 and $88.2 \%$ respectively [26]. However, Erythromycin resistance was found in 51.4\% of isolates in India [27]. In Brazil, resistance to erythromycin and clindamycin was $15.4 \%$ [28]. Thus, antimicrobial susceptibility test is suggested before choosing erythromycin as an alternative treatment for penicillin-allergic patients.

Traditionally, GAS infection patients with penicillin allergy are commonly treated with macrolide antibiotics. In the late 1990s, the resistance rate of GAS isolates to erythromycin in most regions of China was less than $50 \%$. Around 2008, the resistance rate of GAS to erythromycin was $95-100 \%$, while that for the isolates in Taiwan decreased from $53.1 \%$ in 2000 to $0 \%$ in 2010 , but rapidly increased to $65 \%$ in 2011 . The genes involved in erythromycin resistance were mefA $(53.1 \%)$, ermB (35.9\%), and ermTR (10.9\%) [29]. In this study, the resistance rate to erythromycin was $98.3 \%$, that was much higher than that detected in North America and some European countries (9.6-35.8\%). Of the total 297 isolates, $290(97.64 \%)$ harbored the ermB gene, 5 (1.68\%)harbored mefA, none harbored ermA. This phenomenon differed from that in the USA, Italy, Chile, and Canada where erythromycin-resistant strains of GAS are mainly M-resistant phenotypes mediated by $m e f A$. The target modification mechanism mediated by ermB is the main resistance mechanism of GAS in China. The pattern of antibiotic resistance fluctuates worldwide. In a study in India, $51.4 \%$ of the GAS isolates were resistant to erythromycin, of which, $65.1 \%$ harbored $\operatorname{ermB}$ and $32.5 \%$ harbored mefA as the only genes resistant to macrolides, while $2.2 \%$ harbored both ermB and mefA [8]. The resistance rate of erythromycin and clindamycin in Korea decreased from 51.0 and $33.7 \%$ in 2002 to 9.8 and $8.8 \%$ in 2004 , respectively. The sharp decline in erythromycin resistance in a short period may be related to the change in emm type distribution in the community [30]. In Portugal, the resistance rates of erythromycin and clindamycin were 14\% (carrying the erm $\mathrm{B}$ gene) and 9\% (harboring the ermTR gene) in 2010-2015, respectively [31]. Thus, it could be deduced that the high resistance rate of macrolides in China was related to the distribution of emm types.

The distribution of emm genotypes of GAS varied according to the countries, regions, and periods. emm 1 is the most popular type in Germany, consistent with that in the USA, Australia, and Japan; the prevalent types were emm1 (31.8\%), emm28 (15.4\%), and emm 89 $(14.5 \%)[14,32]$. Presently, the most popular emm types in China are emm12 and emm1. In 2011, two patients with scarlet fever died in Hong Kong; the GAS pathogens were emm1 and emm12 [33]. In Chaoyang district, Beijing, in 2011, the main GAS epidemic strain of scarlet fever in children was emm12.0 [34]. In our study, 297 GAS isolates were recovered from patients with scarlet fever at the Children's Hospital from 2016 to 2017. Nine emm types, including 28 subtypes, were identified, of which, emm12 $(65.32 \%, 194 / 297)$ and emm1 (27.61\%, $82 / 297$ ) were the most prevalent emm types (Table 4). In a previous study, eight emm types were identified in 155 isolates of GAS recovered from the pharynx of children with scarlet fever, pharyngeal tonsillitis, as well as healthy carrier in Beijing. emm1.0 and emm12.0 were the main types of scarlet fever and pharyngeal tonsillitis. stg485, emm18.0, emm1.0, and emm12.0 were the main types of healthy carrier [35]. From 2009 to 2016, the 
Table 5 Distribution of emm types erythromycin-resistant genes and superantigens in 297 GAS isolates from Children's Hospital during 2016 to 2017

\begin{tabular}{|c|c|c|c|c|c|c|c|c|c|c|c|c|c|c|c|}
\hline \multicolumn{2}{|l|}{ emm genes } & \multicolumn{3}{|c|}{ erythromycin-resistant genes } & \multicolumn{11}{|c|}{ superantigens } \\
\hline Type & n. & $\begin{array}{l}\text { ermB } \\
(\mathrm{n} / \%)\end{array}$ & $\begin{array}{l}\text { ermA } \\
(\mathrm{n} / \%)\end{array}$ & $\begin{array}{l}\text { mefA } \\
(\mathrm{n} / \%)\end{array}$ & $\begin{array}{l}\text { speA } \\
\text { (n) }\end{array}$ & $\begin{array}{l}\text { spec } \\
\text { (n) }\end{array}$ & $\begin{array}{l}\text { speG } \\
\text { (n) }\end{array}$ & $\begin{array}{l}\text { speH } \\
\text { (n) }\end{array}$ & $\begin{array}{l}\text { spel } \\
\text { (n) }\end{array}$ & $\begin{array}{l}\text { speJ } \\
\text { (n) }\end{array}$ & $\begin{array}{l}\text { spek } \\
\text { (n) }\end{array}$ & $\begin{array}{l}\text { spel } \\
\text { (n) }\end{array}$ & $\begin{array}{l}\text { speM } \\
\text { (n) }\end{array}$ & $\begin{array}{l}\text { ssa } \\
\text { (n) }\end{array}$ & $\begin{array}{l}\text { smeZ } \\
\text { (n) }\end{array}$ \\
\hline emm1.0 & 78 & $78 / 26.3$ & $0 / 0$ & $2 / 0.67$ & 70 & 76 & 74 & 3 & 5 & 55 & 1 & 2 & 13 & 66 & 76 \\
\hline emm12.0 & 139 & $136 / 45.79$ & 0/0 & $1 / 0.34$ & 11 & 125 & 126 & 123 & 124 & 6 & 0 & 3 & 40 & 130 & 134 \\
\hline emm3.1 & 3 & $1 / 0.34$ & $0 / 0$ & $1 / 0.34$ & 2 & 2 & 3 & 0 & 1 & 0 & 3 & 0 & 1 & 1 & 3 \\
\hline emm4.0 & 1 & $1 / 0.34$ & 0/0 & $0 / 0$ & 0 & 1 & 1 & 0 & 0 & 0 & 0 & 0 & 0 & 1 & 1 \\
\hline emm6.19 & 1 & $1 / 0.34$ & 0/0 & $0 / 0$ & 1 & 1 & 1 & 0 & 0 & 0 & 1 & 0 & 1 & 0 & 1 \\
\hline emm6.4 & 1 & $1 / 0.34$ & 0/0 & $0 / 0$ & 1 & 1 & 1 & 0 & 0 & 0 & 1 & 0 & 1 & 0 & 1 \\
\hline emm6.89 & 1 & $1 / 0.34$ & 0/0 & $0 / 0$ & 1 & 1 & 1 & 0 & 0 & 0 & 0 & 0 & 0 & 0 & 1 \\
\hline emm75.0 & 8 & $8 / 2.69$ & 0/0 & $0 / 0$ & 1 & 8 & 7 & 6 & 8 & 1 & 0 & 8 & 6 & 0 & 8 \\
\hline emm89.0 & 4 & $4 / 1.35$ & 0/0 & $0 / 0$ & 0 & 4 & 4 & 0 & 0 & 0 & 0 & 0 & 1 & 0 & 4 \\
\hline emm225 & 1 & $1 / 0.34$ & 0/0 & $0 / 0$ & 1 & 1 & 1 & 0 & 0 & 0 & 0 & 0 & 0 & 1 & 1 \\
\hline stg 485.0 & 1 & $1 / 0.34$ & 0/0 & $0 / 0$ & 0 & 0 & 0 & 0 & 0 & 0 & 0 & 0 & 0 & 0 & 0 \\
\hline emm1.25 & 1 & $1 / 0.34$ & 0/0 & $0 / 0$ & 1 & 1 & 1 & 0 & 0 & 1 & 0 & 0 & 0 & 1 & 1 \\
\hline emm1.3 & 1 & $1 / 0.34$ & 0/0 & $0 / 0$ & 1 & 1 & 1 & 0 & 0 & 1 & 0 & 0 & 0 & 1 & 1 \\
\hline emm 1.33 & 2 & $2 / 0.67$ & 0/0 & $0 / 0$ & 2 & 2 & 2 & 0 & 0 & 2 & 0 & 0 & 0 & 2 & 2 \\
\hline emm12.12 & 1 & $1 / 0.34$ & 0/0 & $0 / 0$ & 0 & 1 & 1 & 1 & 1 & 0 & 0 & 0 & 0 & 1 & 1 \\
\hline emm12.13 & 1 & $1 / 0.34$ & 0/0 & $0 / 0$ & 0 & 0 & 1 & 1 & 1 & 0 & 0 & 1 & 1 & 1 & 1 \\
\hline emm12.19 & 27 & 27/9.09 & 0/0 & $0 / 0$ & 4 & 26 & 25 & 3 & 3 & 0 & 0 & 1 & 4 & 27 & 27 \\
\hline emm12.20 & 1 & $1 / 0.34$ & 0/0 & $1 / 0.34$ & 1 & 1 & 1 & 1 & 1 & 0 & 0 & 0 & 0 & 1 & 1 \\
\hline emm12.21 & 2 & $2 / 0.67$ & 0/0 & 0/0 & 0 & 1 & 1 & 1 & 2 & 0 & 0 & 0 & 0 & 1 & 1 \\
\hline emm12.30 & 1 & $1 / 0.34$ & 0/0 & $0 / 0$ & 0 & 0 & 1 & 1 & 1 & 0 & 0 & 0 & 1 & 0 & 1 \\
\hline emm12.36 & 4 & $4 / 1.35$ & 0/0 & $0 / 0$ & 2 & 4 & 4 & 3 & 3 & 0 & 0 & 0 & 0 & 4 & 4 \\
\hline emm12.37 & 8 & $6 / 2.02$ & 0/0 & $0 / 0$ & 2 & 8 & 7 & 7 & 7 & 0 & 0 & 0 & 2 & 8 & 8 \\
\hline emm12.40 & 2 & $2 / 0.67$ & 0/0 & $0 / 0$ & 0 & 2 & 1 & 2 & 2 & 0 & 0 & 0 & 1 & 2 & 2 \\
\hline emm12.66 & 2 & $2 / 0.67$ & 0/0 & $0 / 0$ & 0 & 2 & 2 & 2 & 2 & 0 & 0 & 0 & 1 & 2 & 2 \\
\hline emm12.69 & 2 & $2 / 0.67$ & 0/0 & $0 / 0$ & 1 & 2 & 1 & 0 & 0 & 0 & 0 & 0 & 0 & 2 & 2 \\
\hline emm12.7 & 1 & $1 / 0.34$ & 0/0 & $0 / 0$ & 0 & 1 & 1 & 0 & 0 & 0 & 0 & 0 & 0 & 0 & 1 \\
\hline emm12.72 & 2 & $2 / 0.67$ & 0/0 & $0 / 0$ & 0 & 2 & 2 & 0 & 0 & 0 & 0 & 0 & 0 & 2 & 2 \\
\hline emm12.76 & 1 & $1 / 0.34$ & 0/0 & $0 / 0$ & 0 & 1 & 1 & 1 & 1 & 0 & 0 & 0 & 0 & 0 & 1 \\
\hline Percentage(\%) & & 97.64 & 0 & 1.68 & 34.34 & 92.59 & 91.58 & 52.19 & 54.55 & 22.22 & 2.02 & 5.05 & 24.58 & 85.52 & 96.97 \\
\hline
\end{tabular}

main emm types of GAS strains were emm12 (42.9$62.2 \%)$ and emm1 (30.7-35.0\%) [25, 36]. Interestingly, the proportion of emm12 and emm1 in this study was similar to that reported previously. These results showed that the emm genotypes of GAS isolates changed significantly in recent years as compared to those identified in the 1990s. The most common emm genotypes in 19931994 were emm3.1, emm1.0, emm4.0, emm12.0, st1815.0, emm6.0, and emm18.0 [37]. You Y collected 2484 strains of GAS during 2011-2018 and found that the prevalent emm types of GAS causing scarlet fever shifted for 8 years in Beijing since 2012, the frequency of emm12 S pyogenes started to decline from 2011, whereas emm1 started to increase and then exceeded emm12 in
2013 and 2014. Since 2015, emm12 exceeded emm1 and became the main type again. Notably, numbers of nonpredominant types emm128 increased substantially in 2017 and of emm3 in 2018 [38].

The main types of GAS in China are different from those in other countries around the world. A total of 35 emm types in 1282 isolates from GAS infection in children in Greece from 2007 to 2013, included emm1 (16.7\%), emm12 (13.6\%), emm77 (10.9\%), emm6 (6.8\%), and emm89 (6.6\%) [1]. Among 1122 invasive isolates from Finland during 2008-2013, $72 \mathrm{emm}$ types were identified, of which emm28 (26\%), emm89 (12\%), and emm1 (12\%) were the most common types [39]. The main emm types of iGAS strains in Portugal from 2010 
to 2015 were emm1 (28\%), emm89 (11\%), emm3 (9\%), emm12 (8\%), and emm6 (7\%) [31]. Furthermore, the isolates of emm60.1 and emm63.0 genotypes were prevalent in the children from the villages of Guizhou Province in China, which led to the outbreak of acute glomerulonephritis in 2005 [40]. In 2012, many people suddenly had a fever, sore throat and/or fatigue, headache, and other similar symptoms within $24 \mathrm{~h}$ in Beijing. The isolated GAS strain had the same genotype (emm89), which was first discovered to cause tonsillar pharyngitis in Beijing, China [41]. emm89was also identified in this study. Between January 2016 and May 2017, a rare outbreak of GAS, caused by emm66.0, occurred in England and Welsh [42]. The local outbreak of GAS infection is related to the shift of emm types. Moreover, different emm types carry different resistance genes, which leads to the difference of erythromycin resistance rate. In erythromycinresistance isolates in Brazil the ermB gene was predominant, followed by the ermA gene. Thirty-two emm types and subtypes were found, but five (emm1, emm4, emm12, emm22, emm81) were detected in $48 \%$ of the isolates [28]. These results were different from that in China [21]. Therefore, continuous monitoring of streptococcal infection is required.

GAS superantigens, except speG, speJ, and smeZ encoded by chromosome, speA, speC, speH, speI, speK, speL, speM, and ssa are encoded by phage, which is the main driving force for pathogenic strains to obtain pathogenic factors through transfer. The transfer and mutation of genes can produce highly pathogenic GAS strains, which affect the epidemic situation of the GAS disease, resulting in different distributions of the $S$. pyogenes superantigen gene spectrum in different periods and geographical areas. A study from Portugal showed that smeZ (96.0\%) and speG (86.9\%) were common in GAS, followed by speC, ssa, speJ, speA, speK, and speI [43]. A multicenter study in China has proved that $31.1 \%$ of the GAS isolates harbored speA, while $58.6 \%$ harbored speC [17]. emm1 and emm12 were consistently the most prevalent types of GAS isolates from pediatric patients during 1993-1994 and 2005-2006. Isolates carrying six or more superantigen genes increased from $46.53 \%$ in $1993-1994$ to $78.39 \%$ in $2005-2006$. The level of $s s a, s p e H$, and speJ genes increased, while that of speA decreased. The gene profiles of superantigen were associated with the emm type, but strains of the same emm type occasionally carry different superantigen genes in the two periods. Intriguingly, no significant difference was detected in the distribution of emm types and $S A g$ gene profile between isolates from different diseases [37]. In this study, 11 superantigens were detected in GAS isolates, and speC, speG, and smeZ were the most common superantigens. emm1 harbored speA, speC, speG, speJ, speM, ssa, and smeZ, but the content of speI, speK, speL was less. emm12 type tended to contain speC, speG, speH, speI, speM, ssa, and smeZ, with little or no speJ, speK, and speL. A study from Germany showed that the most common superantigen genes in GAS were speG (92.1\%), speJ (50.9\%), and speC (42.0\%). Simultaneously, it was observed that emm types or superantigen genes had significant correlation with clinical complications [14]. In an outbreak of GAS infection caused by a rare emm58 type in a multiple trauma treatment center, it was found that the strain was macrolide and tetracycline resistant and produced the Streptococcal exotoxins speB (a streptococcal cysteine protease), speC, speG, smeZ and speF (now considered to be a DNase) [44]. From 2009 to 2016, all isolates from infected patients in 10 general tertiary hospitals in 7 provinces (cities) of China, whether invasive or no-invasive, harbored genes for the protease speB and the pore-forming toxin slo. The other virulence genes, smeZ, speF, and speC accounted for 96.4, 91.4, and $87.1 \%$ of collected isolates, respectively. All strains were sensitive to penicillin, ampicillin, cefotaxime, and vancomycin, whereas the resistance rates to erythromycin, clindamycin, and tetracycline were 93.5, 94.2, and $86.4 \%$ respectively, which indicated high genotype diversity and high prevalence of macrolide resistance of $S$. pyogenes in clinical isolates circulating in China [25]. In the previous studies on children, 30.5 and $57.2 \%$ of GAS isolates harbored superantigen genes speA and speC, respectively. $88.8 \%$ of emm1.0 genotype strains contained the speA gene, while $69.6 \%$ of the emm12.0 genotype strains contained the speA gene [17]. In Taiwan, isolates with emm1.0, emm4.0, and emm12.0 genotype were the main causes of noninvasive diseases. A few isolates with emm1.0 genotype harbored speC and speH genes, while a few isolates with emm12.0 genotype harbored speJ and $s m e Z$ genes [45]. In Spain, the isolates of s.pyogenes with emm1.0 genotype, associated with pharyngitis, carried speA, spe G and speJ genes, but did not carry speC, speH, speI or ssa genes [46]. All the above studies showed that the distribution of emm genotypes and superantigen gene profiles were time and region dependent.

\section{Conclusions}

The classification of pathogenic microorganisms is essential for epidemiological research. In this study, all GAS isolates from the Children's Hospital were sensitive to penicillin, ceftriaxone, and vancomycin, and highly resistant to erythromycin and clindamycin. Nevertheless, penicillin can still be used as the first option for the 
treatment of streptococcal infections. Emm gene typing study shows that $e m m 12$ and $e m m 1$ are the most prevalent genetypes. ermB gene is a key role of Streptococcus in the resistance to macrolides, and speC, speG, and sme $Z$ are the most common superantigens of GAS. The long-term monitoring of the emm types and superantigens of GAS is crucial for understanding the variations of GAS M protein, the generation of new bacterial type, the prediction of epidemic trends and the preparation of vaccine.

\section{Abbreviations}

GAS: Group A streptococcus; emm: Encoding mature M Protein gene; CLSI: Clinical and Laboratory Standards Institute; MIC: Minimum inhibitory concentration; CDC: Center for Disease Control and Prevention PCR: Polymerase chain reaction

\section{Acknowledgements}

Not applicable.

\section{Authors' contributions}

$L H, Z L, Z Y$ designed the study; LH, ZL, LX, HJ, and ML collected data; LX, HJ, and $M L$ coordinated and supervised the data collection; $Z Y, L H$, and $Z L$ analyzed the data; LH and ZL participated in the interpretation of data; $L H$ and $Z Y$ drafted the initial manuscript. All authors approved the final manuscript as submitted and agree to be accountable for all aspects of work.

\section{Funding}

This work was supported by Research Foundation of Capital Institute of Pediatrics (NO.QN-2016-09) and Research Foundation of Capital Institute of Pediatrics (NO.PY-2020-05). The study sponsors had no role in study design; collection, analysis, and interpretation of data; writing the report; or the decision to submit the report for publication.

\section{Availability of data and materials}

The datasets generated and/or analyzed during the current study are available from the corresponding author on reasonable request.

\section{Ethics approval and consent to participate}

This study was approved by the Ethics Committee of the Capital Institute of Pediatrics. Written consent forms were obtained from the participants' guardians before collecting the pharyngeal swab samples, and the anonymity of the participants was guaranteed.

\section{Consent for publication}

Not applicable.

\section{Competing interests}

The authors have indicated they have no competing interests.

\section{Author details}

'Department of Dermatology, Children's Hospital Affiliated to Capital Institute of Pediatrics, Beijing 100020, China. ${ }^{2}$ Department of Clinical Laboratory, Children's Hospital Affiliated to Capital Institute of Pediatrics, Beijing 100020, China. ${ }^{3}$ The Sixth Medical Centre of PLA, General Hospital, Beijing 100048, China.

Received: 23 January 2020 Accepted: 7 July 2020

Published online: 13 July 2020

\section{References}

1. Koutouzi F, Tsakris A, Chatzichristou P, Koutouzis E, Daikos GL, Kirikou E, Petropoulou N, Syriopoulou V, Michos A. Streptococcus pyogenes emm types and clusters during a 7-year period (2007 to 2013) in pharyngeal and nonpharyngeal pediatric isolates. J Clin Microbiol. 2015;53(7):2015-21.

2. Carapetis JR, Steer AC, Mulholland EK, Weber M. The global burden of group a streptococcal diseases. Lancet Infect Dis. 2005;5(11):685-94.
3. Zheng PX, Chan YC, Chiou CS, Chiang-Ni C, Wang SY, Tsai PJ, Chuang WJ, Lin YS, Liu CC, Wu JJ. Clustered regularly interspaced short palindromic repeats are emm type-specific in highly prevalent group a streptococci. PLoS One. 2015;10(12):e0145223.

4. Wu S, Peng X, Yang Z, Ma C, Zhang D, Wang Q, Yang P. Estimated burden of group a streptococcal pharyngitis among children in Beijing, China. BMC Infect Dis. 2016;16(1):452.

5. You YH, Song YY, Yan XM, Wang HB, Zhang MH, Tao XX, Li LL, Zhang YX, Jiang XH, Zhang BH, et al. Molecular epidemiological characteristics of Streptococcus pyogenes strains involved in an outbreak of scarlet fever in China, 2011. Biomed Environ Sci. 2013;26(11):877-85.

6. Tse H, Bao JY, Davies MR, Maamary P, Tsoi HW, Tong AH, Ho TC, Lin CH, Gillen CM, Barnett TC, et al. Molecular characterization of the 2011 Hong Kong scarlet fever outbreak. J Infect Dis. 2012;206(3):341-51.

7. Grivea IN, Al-Lahham A, Katopodis GD, Syrogiannopoulos GA, Reinert RR. Resistance to erythromycin and telithromycin in Streptococcus pyogenes isolates obtained between 1999 and 2002 from Greek children with tonsillopharyngitis: phenotypic and genotypic analysis. Antimicrob Agents Chemother. 2006;50(1):256-61.

8. Abraham T, Sistla S. Molecular epidemiology of macrolide resistant group a streptococci from Puducherry, India. J Infect Dev Ctries. 2017:11(9):679-83.

9. Li HX, Zhou L, XU J, Cao HN, Zhang HL, Ma YC, Liu XY, Hu J. Application of group a streptococcal antigen detection and drug sensitivity analysis. Bei Jing Yi Xue. 2019;41(11):1010-2.

10. Cui JH, Miao Y, Hu XF, Jin B, Ji YL, Li D. Genotyping of emm gene in group a hemolytic streptococcus from children in Xicheng district of Beijing from 2011 to 2013. Chin J Health Lab Tec. 2014;24(14):2048-50.

11. McMillan DJ, Sanderson-Smith ML, Smeesters PR, Sriprakash KS. Molecular markers for the study of streptococcal epidemiology. Curr Top Microbiol Immunol. 2013;368:29-48.

12. Bessen DE, Smeesters PR, Beall BW: Molecular Epidemiology, Ecology, and Evolution of Group A Streptococci. Microbiol Spectr 2018, 6(5).

13. Bley C, van der Linden M. Reinert RR: mef(a) is the predominant macrolide resistance determinant in Streptococcus pneumoniae and Streptococcus pyogenes in Germany. Int J Antimicrob Agents. 2011;37(5):425-31.

14. Imohl M. Fitzner C, Perniciaro $S$, van der Linden M. Epidemiology and distribution of 10 superantigens among invasive Streptococcus pyogenes disease in Germany from 2009 to 2014. PLoS One. 2017:12(7):e0180757.

15. Shannon BA, McCormick JK, Schlievert PM: Toxins and Superantigens of Group A Streptococci. Microbiol Spectr 2019, 7(1).

16. Jing $H B$, Ning $B A$, Hao $H J$, Zheng $Y L$, Chang $D$, Jiang W, Jiang YQ. Epidemiological analysis of group a streptococci recovered from patients in China. J Med Microbiol. 2006;55(Pt 8):1101-7.

17. Liang Y, Liu X, Chang H, Ji L, Huang G, Fu Z, Zheng Y, Wang L, Li C, Shen Y, et al. Epidemiological and molecular characteristics of clinical isolates of Streptococcus pyogenes collected between 2005 and 2008 from Chinese children. J Med Microbiol. 2012;61(Pt 7):975-83.

18. CLSI. Performance standards for antimicrobial susceptibility testing: clinical and laboratory standards institute (CLSI), M100. 28th ed; 2018.

19. Perez-Trallero E, Montes M, Orden B, Tamayo E, Garcia-Arenzana JM, Marimon JM. Phenotypic and genotypic characterization of Streptococcus pyogenes isolates displaying the MLSB phenotype of macrolide resistance in Spain, 1999 to 2005. Antimicrob Agents Chemother. 2007;51(4):1228-33.

20. Green NM, Beres SB, Graviss EA, Allison JE, McGeer AJ, Vuopio-Varkila J, LeFebvre RB, Musser JM. Genetic diversity among type emm28 group a Streptococcus strains causing invasive infections and pharyngitis. J Clin Microbiol. 2005:43(8):4083-91.

21. You Y, Davies MR, Protani M, McIntyre L, Walker MJ, Zhang J. Scarlet fever epidemic in China caused by Streptococcus pyogenes serotype M12: epidemiologic and molecular analysis. EBioMedicine. 2018;28:128-35.

22. Lamagni T, Guy R, Chand M, Henderson KL, Chalker V, Lewis J, Saliba V, Elliot AJ, Smith GE, Rushton $S$, et al. Resurgence of scarlet fever in England, 2014-16: a population-based surveillance study. Lancet Infect Dis. 2018;18(2):180-7.

23. Liu Y, Chan TC, Yap LW, Luo Y, Xu W, Qin S, Zhao N, Yu Z, Geng X, Liu SL. Resurgence of scarlet fever in China: a 13-year population-based surveillance study. Lancet Infect Dis. 2018;18(8):903-12.

24. DeMuri GP, Sterkel AK, Kubica PA, Duster MN, Reed KD, Wald ER. Macrolide and clindamycin resistance in group a streptococci isolated from children with pharyngitis. Pediatr Infect Dis J. 2017;36(3):342-4. 
25. Lu B, Fang Y, Fan Y, Chen X, Wang J, Zeng J, Li Y, Zhang Z, Huang L, Li H, et al. High prevalence of macrolide-resistance and molecular characterization of Streptococcus pyogenes isolates circulating in China from 2009 to 2016. Front Microbiol. 2017:8:1052.

26. Zhao CJ, Peng XM, Yang P, Shi WX, Lu GL, Pang Y. Research on the drug sensitivity test results of group a Streptococcus in children in Beijing in 2013. Chinese Journal of Health Laboratory Technology. 2015;25(15):2623-5.

27. Abraham T, Sistla S. Trends in antimicrobial resistance patterns of group a streptococci, molecular basis and implications. Indian J Med Microbiol. 2018; 36(2):186-91.

28. Areas GP, Schuab RB, Neves FP, Barros RR. Antimicrobial susceptibility patterns, emm type distribution and genetic diversity of Streptococcus pyogenes recovered in Brazil. Mem Inst Oswaldo Cruz. 2014;109(7):935-9.

29. Chuang PK, Wang SM, Lin HC, Cho YH, Ma YJ, Ho TS, Shen CF, Liu CC. The trend of macrolide resistance and emm types of group a streptococci from children at a medical center in southern Taiwan. J Microbiol Immunol Infect. 2015;48(2):160-7.

30. Koh EH, Kim S, Lee NY. Decrease of erythromycin resistance in group a streptococci by change of emm distribution. Jpn J Infect Dis. 2008;61(4): 261-3.

31. Friaes A, Melo-Cristino J, Ramirez M. Changes in emm types and superantigen gene content of Streptococcus pyogenes causing invasive infections in Portugal. Sci Rep. 2019;9(1):18051

32. Gherardi G, Vitali LA, Creti R. Prevalent emm types among invasive GAS in Europe and North America since year 2000. Front Public Health. 2018;6:59.

33. Hsieh YC, Huang YC. Scarlet fever outbreak in Hong Kong, 2011. J Microbiol Immunol Infect. 2011;44(6):409-11.

34. Wang HB, Song YY, You YH, Wang HW, Han QH, Zhao JH, Zhang XX. Molecular epidemiological analysis of group a streptococci isolated from children in Chaoyang District of Beijing, 2011: emm types, virulence factor genes and erythromycin resistant genes. Biomed Environ Sci. 2013;26(9): 782-4.

35. Liang YM, Chang HS, Shen XZ, Yu SJ, Yuan L, Yang YH. Relationship between emm typing and Superantigen genes speA and speC of Streptococcus Pyogenes isolated from children in Beijing. Journal of Applied Clinical Pediatrics. 2010;25(22):1700-2.

36. Zhang DT, Yang P, Wu SS, Zhao JC, Lu GL, Guo J, Wang QY, Peng XM. M protein gene of group a streptococcus isolated from children with pharyngeal infection in Beijing, 2011-2014. Disease Surveillance. 2015;30(11): 917-21.

37. Ma $Y$, Yang $Y$, Huang $M$, Wang $Y$, Chen $Y$, Deng L, Yu S, Deng $Q$, Zhang $H$, Wang $C$, et al. Characterization of emm types and superantigens of Streptococcus pyogenes isolates from children during two sampling periods. Epidemiol Infect. 2009:137(10):1414-9.

38. You Y, Peng X, Yang P, Wang Q, Zhang J. 8-year M type surveillance of Streptococcus pyogenes in China. Lancet Infect Dis. 2020;20(1):24-5

39. Smit PW, Lindholm L, Lyytikainen O, Jalava J, Patari-Sampo A, Vuopio J. Epidemiology and emm types of invasive group a streptococcal infections in Finland, 2008-2013. Eur J Clin Microbiol Infect Dis. 2015;34(10):2131-6.

40. Zheng MH, Jiao ZQ, Zhang LJ, Yu SJ, Tang GP, Yan XM, He LH, Meng FL, Zhao F, Zhang MJ, et al. Genetic analysis of group a streptococcus isolates recovered during acute glomerulonephritis outbreaks in Guizhou Province of China. J Clin Microbiol. 2009:47(3):715-20.

41. Liu YM, Zhao JZ, Li BB, Yang JY, Dong XG, Zhang JJ, Cao B. A report on the first outbreak of a single clone group a Streptococcus (emm-type 89) tonsillopharyngitis in China. J Microbiol Immunol Infect. 2014;47(6):542-5.

42. Bubba L, Bundle N, Kapatai G, Daniel R, Balasegaram S, Anderson C, Chalker V, Lamagni T, Brown C, Ready D, et al. Genomic sequencing of a national emm66 group a streptococci (GAS) outbreak among people who inject drugs and the homeless community in England and Wales, January 2016may 2017. J Inf Secur. 2019;79(5):435-43.

43. Friaes A, Pinto FR, Silva-Costa C, Ramirez M, Melo-Cristino J. Group a streptococci clones associated with invasive infections and pharyngitis in Portugal present differences in emm types, superantigen gene content and antimicrobial resistance. BMC Microbiol. 2012;12:280.

44. Mathur P, Bhardwaj N, Gupta G, Punia P, Tak V, John NV, Agrawal D, Misra MC. Outbreak of Streptococcus pyogenes emm type 58 in a high dependency unit of a level-1 trauma center of India. Indian J Crit Care Med. 2014;18(2):77-82.
45. Lin HC, Wang SM, Lin YL, Lin YS, Wu JJ, Chuang WJ, Lin MT, Liu CC. Group a streptococcal infection caused by emm1 strains among children in southern Taiwan. Eur J Clin Microbiol Infect Dis. 2008;27(12):1253-6.

46. Rivera A, Rebollo M, Miro E, Mateo M, Navarro F, Gurgui M, Mirelis B, Coll P. Superantigen gene profile, emm type and antibiotic resistance genes among group a streptococcal isolates from Barcelona, Spain. J Med Microbiol. 2006;55(Pt 8):1115-23.

\section{Publisher's Note}

Springer Nature remains neutral with regard to jurisdictional claims in published maps and institutional affiliations.
Ready to submit your research? Choose BMC and benefit from:

- fast, convenient online submission

- thorough peer review by experienced researchers in your field

- rapid publication on acceptance

- support for research data, including large and complex data types

- gold Open Access which fosters wider collaboration and increased citations

- maximum visibility for your research: over $100 \mathrm{M}$ website views per year

At BMC, research is always in progress.

Learn more biomedcentral.com/submissions 